\title{
Asymptotic behaviour of solutions of a nonlinear transport equation
}

By C.J.van Duijn and M.A. Peletier at Amsterdam

\section{Introduction}

In this paper we consider the asymptotic behaviour as $t \rightarrow \infty$ and as $\varepsilon \downarrow 0$ of radial solutions of the equation

$$
\beta(u)_{t}+\operatorname{div}\left(u \frac{\lambda}{|x|} \mathbf{e}_{r}-\nabla u\right)=0 \text { for }(x, t) \in \Omega_{\varepsilon} \times \mathbb{R}^{+},
$$

satisfying the boundary condition

$$
\frac{\partial u}{\partial v}=\frac{\lambda}{\varepsilon}\left(u_{e}-u\right) \quad \text { for }(x, t) \in \partial \Omega_{\varepsilon} \times \mathbb{R}^{+} .
$$

The set $\Omega_{\varepsilon}$ is the outer domain $\left\{x \in \mathbb{R}^{2}:|x|>\varepsilon\right\}, \mathrm{e}_{r}$ is the unit vector in the radial direction, $\lambda>0$ and $u_{e}$ are given constants, and $\beta:[0, \infty) \rightarrow[0, \infty)$ is a function to be specified later. We use the subscript notation to denote partial derivatives.

Problem (1.1-2) arises in a two-dimensional model describing the transport of reactive solutes, with scaled concentration $u$, through a porous medium in which the groundwater flow is induced by well injection. The small parameter $\varepsilon$ is related to the well radius and the parameter $\lambda$ is the Peclet number. The boundary condition (1.2) describes the injection of water with solute concentration $u_{e}$ into the flow domain.

The nonlinear function $\beta$ in equation (1.1) reflects the effect of equilibrium adsorption reactions of the dissolved chemicals on the surface of the soil particles. The canonical example is $\beta(u)=u+\psi(u)$, where $\psi$ is the so-called adsorption isotherm [4]. Two typical isotherms are the Langmuir isotherm

$$
\psi(u)=\frac{k_{1} u}{1+k_{2} u}, \quad k_{1}, k_{2}>0,
$$

and the Freundlich isotherm 


$$
\psi(u)=k u^{p}, \quad k>0,0<p<1
$$

This model is described in detail by van Duijn and Knabner in [5]; we refer to [4] for a general discussion of the underlying physical and chemical assumptions.

Since we only consider radial solutions, we seek a function $u=u(r, t)$ that satisfies

$$
\mathrm{I} \begin{cases}\beta(u)_{t}+\frac{\lambda-1}{r} u_{r}-u_{r r}=0, & \varepsilon<r<\infty, t>0, \\ u_{r}=\frac{\lambda}{\varepsilon}\left(u-u_{e}\right), & r=\varepsilon, t>0, \\ u(r, 0)=u_{0}(r), & r>\varepsilon,\end{cases}
$$

where $u_{0}:[\varepsilon, \infty) \rightarrow[0, \infty)$ denotes the radially symmetric initial distribution. Without loss of generality we may consider the cases

$$
\text { contamination process: } u_{e}=1, u_{0}(\infty)=0 \text {, }
$$

and

$$
\text { remediation process: } u_{e}=0, u_{0}(\infty)=1 \text {, }
$$

where we suppose that $u_{0}(\infty):=\lim _{r \rightarrow \infty} u_{0}(r)$ exists. Furthermore we suppose that $u_{0}$ satisfies

(A 1) $u_{0} \in C^{0,1}([\varepsilon, \infty)), 0 \leqq u_{0} \leqq 1$, and $r u_{0}^{\prime}(r)$ is uniformly bounded on $(\varepsilon, \infty)$.

About the function $\beta$ we assume (cf. [4]):

$$
\begin{aligned}
& \left(\mathrm{H}_{\beta} 1\right) \quad \beta \in C^{\infty}(0, \infty) \cap C([0, \infty)) ; \\
& \left(\mathrm{H}_{\beta} 2\right) \quad \beta(0)=0, \beta^{\prime}(s)>0 \text { and } \beta^{\prime \prime}(s) \leqq 0 \text { for } s>0 .
\end{aligned}
$$

Note that since $\beta^{\prime}(0+)=\infty$ is allowed by Hypotheses $\left(\mathrm{H}_{\beta} 1-2\right)$, our formulation must include this degenerate case. One of the consequences is the existence of free boundaries separating the regions where $u>0$ from the regions where $u=0$. Furthermore we note that equation (I a) with $\lambda=1$ reduces to the well-known porous media equation

$$
v_{t}=\phi(v)_{r r} \text { with } \phi=\beta^{-1} .
$$

A Cauchy-Dirichlet problem for equation (I a) with $\lambda>1$ was studied by Goncerzewicz [10], generalising results by Gilding [8] and Díaz and Kersner [3] who considered general convection-diffusion equations in $\mathbb{R}^{1}$. Following these authors we introduce weak solutions in the following sense. Let $T$ be some fixed positive number, which eventually will tend to infinity, and consider the half strip $S_{T}^{\varepsilon}=\{(r, t): \varepsilon<r<\infty, 0<t<T\}$.

Definition. A non-negative function $u: \overline{S_{T}^{\varepsilon}} \rightarrow \mathbb{R}$ is called a weak solution of Problem I if 
(i) $u \in C\left(\overline{S_{T}^{\varepsilon}}\right)$ and $u$ has a bounded weak derivative $u_{r}$ in $S_{T}^{\varepsilon}$;

(ii) for every test function $\varphi \in H^{1}\left(S_{T}^{\varepsilon}\right)$ that vanishes for large $r$ and at $t=T$,

(1.6) $\int_{S_{T}^{\varepsilon}}\left\{\beta(u) \varphi_{r} r+\left(\lambda u-r u_{r}\right) \varphi_{r}\right\} d r d t+\int_{\varepsilon}^{\infty} \beta\left(u_{0}(r)\right) \varphi(r, 0) r d r+\lambda u_{e} \int_{0}^{T} \varphi(\varepsilon, t) d t=0$.

If $u$ satisfies (1.6) with the equality replaced by $\geqq(\leqq)$ and with $\varphi \geqq 0$ in $S_{T}^{\varepsilon}$ then we call $u$ a sub(super)solution.

Hypotheses $\left(\mathrm{H}_{\beta} 1-2\right)$ and (A1) ensure the existence of a unique weak solution $u$ which is smooth in the set $\left\{(r, t) \in S_{T}^{\varepsilon}: u(r, t)>0\right\}$. This is proved in Section 2 .

Remark. Observe that when (I a) is interpreted as a convection-diffusion equation in $\mathbb{R}^{1}$, the sign of $\lambda-1$ determines the direction of the convection: when $\lambda<1$ it is directed towards the origin, and when $\lambda>1$ away from the origin. This distinction will turn out to be important when studying the asymptotic behaviour as $\varepsilon \downarrow 0$.

Our aim is to show that under certain conditions solutions of Problem I converge to self-similar solutions when either $\varepsilon \downarrow 0$ or $t \rightarrow \infty$. The combination of these two limit processes is explained by the following transformation:

$$
\xi=\frac{r}{\varepsilon}, \quad \tau=\frac{t}{\varepsilon^{2}}
$$

under which Problem I becomes

$$
\text { II } \begin{cases}\beta(u)_{\tau}+\frac{\lambda-1}{\xi} u_{\xi}-u_{\xi \xi}=0, & \xi>1, \tau>0, \\ u_{\xi}=\lambda\left(u-u_{e}\right), & \xi=1, \tau>0, \\ u(\xi, 0)=u_{0}(\varepsilon \xi), & \xi>1 .\end{cases}
$$

Obviously the behaviour of solutions of Problem II for large $\tau$ is strongly linked to that of solutions of Problem I for either $\varepsilon \downarrow 0$ or $t \rightarrow \infty$.

A scaling argument leads us to investigate self-similar solutions of equation (Ia) of the form

$$
u(r, t)=f\left(\frac{r}{\sqrt{t}}\right)=f(\eta)
$$

satisfying the equation

$$
\frac{1}{2} \eta^{2}\{\beta(f)\}^{\prime}+\left(\eta f^{\prime}-\lambda f\right)^{\prime}=0,
$$

where primes denote differentiation with respect to $\eta$. Since these self-similar solutions are expected to arise in the limit $\varepsilon \downarrow 0$, we solve equation (1.8) in the domain $0<\eta<\infty$ with the combinations (1.3) and (1.4) as boundary conditions: 

contamination process: $f(0)=1, f(\infty)=0$,

$$
\text { remediation process: } f(0)=0, f(\infty)=1 \text {. }
$$

Note that $\eta=r / \sqrt{t}=\xi / \sqrt{\tau}$, and therefore the self-similar solution satisfies both equation (Ia) and equation (II a).

The boundary value problems (1.8)-(1.9) and (1.8)-(1.10) are studied in Section 3. In Section 4 we prove the main results of this paper. They concern the asymptotic behaviour of weak solutions of Problem I. We shall need an additional hypothesis on $u_{0}$ and $\beta$ in order to prove these results:

$$
\left|\int_{\varepsilon}^{\infty} r\left\{\beta\left(u_{0}(r)\right)-\beta\left(u_{0}(\infty)\right)\right\} d r\right|<\infty
$$

Hypothesis (A 2) can be interpreted physically as stating that the perturbation $u_{0}-u_{0}(\infty)$ of the constant state $u_{0}(\infty)$ has finite mass. We will show in Section 2 that (A2) implies that

$$
\left|\int_{\varepsilon}^{\infty} r\left\{\beta(u(r, t))-\beta\left(u_{0}(\infty)\right)\right\} d r\right|<\infty
$$

for all $t>0$.

The double degeneration of (1.8) with (1.10) - the degeneration of $\beta(f)$ at $f=0$ and the degeneration of the equation at $\eta=0$, which coincide - forces us to assume a technical hypothesis in order to prove the result for the remediation process:

$$
\left(\mathrm{H}_{\beta} 3\right) \quad \lim _{s \downarrow 0} \frac{s \beta^{\prime \prime}(s)}{\beta^{\prime}(s)}=p-1 \quad \text { where } 0<p \leqq 1 .
$$

Note that in the case of a Freundlich isotherm the condition $\left(\mathrm{H}_{\beta} 3\right)$ is satisfied with $0<p<1$, and in the case of a Langmuir isotherm with $p=1$.

The precise asymptotic statements are:

Theorem A. Let hypotheses $\left(\mathrm{H}_{\beta} 1-2\right)$ and (A1-2) be satisfied. Further let $u$ be the solution of Problem I with $u_{e}=1$ and $u_{0}(\infty)=0$ (contamination process), and let $f$ denote the solution of (1.8) and (1.9).

(a) If $\varepsilon$ is fixed, then

$$
\sup _{\varepsilon \leqq r<\infty}|u(r, t)-f(r / \sqrt{t})|=O\left(t^{-k}\right) \quad \text { as } t \rightarrow \infty .
$$

(b) If $u_{0} \equiv 0$, then for fixed $t>0$

$$
\sup _{\varepsilon \leqq r<\infty}|u(r, t)-f(r / \sqrt{t})|=O\left(\frac{\varepsilon^{2}}{t}\right)^{k} \text { as } \varepsilon \rightarrow 0 .
$$

Here the exponent $k$ is given by 


$$
k= \begin{cases}\lambda / 3 & \text { for } \lambda<1 \\ 1 / 3 & \text { for } \lambda \geqq 1\end{cases}
$$

Theorem B. Let hypotheses $\left(\mathrm{H}_{\beta} 1-3\right)$ and (A1-2) be satisfied. Further let $u$ be the solution of Problem I with $u_{e}=0$ and $u_{0}(\infty)=1$ (remediation process), and let $f$ denote the solution of (1.8) and (1.10). Then the conclusions are the same as those of Theorem $\mathrm{A}$ (with $u_{0} \equiv 1$ in part (b)).

Remark. The restriction to constant $u_{0}$ when $\varepsilon$ is varied is a natural one. Since the influence of changes in $\varepsilon$ on the solution is small at a fixed time and away from the well, it is necessary for convergence to self-similar solutions that the initial behaviour of the general solution corresponds to the initial behaviour of the self-similar solution. In practical terms, this means $u_{0}$ has to be constant. Observe that when $u_{0}$ is constant, the two limit processes $\varepsilon \downarrow 0$ and $t \rightarrow \infty$ are truly equivalent.

Remark. As a by-product of the proof of Theorems A and B we obtain a pointwise estimate of $u$. In the contamination case the self-similar solution is a subsolution for the general solution, which implies the following inequality:

$$
0 \leqq 1-u(r, t) \leqq 1-f(r / \sqrt{t}) \text { for all } r>\varepsilon, t>0 .
$$

The behaviour of $1-f(\eta)$ near $\eta=0$ is shown to be proportional to $\eta^{\lambda}$ (Proposition 3.6), and therefore for fixed $r>\varepsilon$

$$
1-u(r, t)=O\left(t^{-\lambda / 2}\right) \text { as } t \rightarrow \infty .
$$

In the same way an estimate follows for the remediation case:

$$
0 \leqq u(r, t) \leqq f(r / \sqrt{t}) \text { for all } r>\varepsilon, t>0
$$

Here the behaviour of $f$ (Proposition 3.9) translates in a similar way to the behaviour of $u(r, t)$ for fixed $r$ as $t$ tends to infinity.

\section{Weak solutions: existence and uniqueness}

We present here the existence and uniqueness results for weak solutions of Problem I. Most of these results are obtained by a straightforward generalisation of the work of Diaz and Kersner [3], Gilding [8], and Goncerzewicz [10]. In those cases we omit the details and only give the appropriate references. However, special attention has to be given to the flux boundary condition at $r=\varepsilon$.

As usual weak solutions are obtained as limits of approximating positive classical solutions. Since these approximations are used later on in this paper to prove the asymptotic results, we describe the procedure in some detail in the existence proof below.

Theorem 2.1. Let hypotheses $\left(\mathrm{H}_{\beta} 1-2\right)$ and (A1) be satisfied. Then Problem I has a unique weak solution. 
Proof. To show existence, we slightly alter the initial and boundary conditions in Problem I, ensuring that the corresponding solution remains strictly positive. This is achieved by considering approximating sequences $\left\{u_{0 n}\right\}_{n \geqq 1}$ and $\left\{u_{e n}\right\}_{n \geqq 1}$, satisfying

$$
\begin{aligned}
& u_{0 n} \in C^{\infty}([\varepsilon, n]) \\
& u_{0 n} \downarrow u_{0} \text { as } n \rightarrow \infty, \text { uniformly on bounded subsets of }[\varepsilon, \infty) \\
& \frac{1}{n} \leqq u_{0 n} \leqq 1 \text { on }[\varepsilon, n] \\
& \sup _{\varepsilon \leqq r \leqq n}\left|r u_{0 n}^{\prime}(r)\right| \leqq \sup _{\varepsilon \leqq r<\infty}\left|r u_{0}^{\prime}(r)\right| \\
& u_{0 n}(r)=\delta_{n} \text { for } n-1 \leqq r \leqq n \\
& u_{0 n}^{\prime}(\varepsilon)=0
\end{aligned}
$$

and

$$
u_{e n}(t)=u_{e}-\left(u_{e}-u_{0 n}(\varepsilon)\right) e^{-n t} \text { for } 0 \leqq t \leqq T,
$$

where the constants $\delta_{n}$ are chosen in $[1 / n, 1]$. Note that the compatibility condition

$$
u_{e n}(0)=u_{0 n}(\varepsilon)
$$

is satisfied. In Problem I we now replace $u_{0}$ by $u_{0 n}$ and $u_{e}$ by $u_{e n}$. This yields the approximate problems

$$
\mathrm{I}_{n} \begin{cases}\beta(u)_{t}+\frac{\lambda-1}{r} u_{r}-u_{r r}=0 & \text { in } S_{T}^{\varepsilon, n}=(\varepsilon, n) \times(0, T], \\ u_{r}=\frac{\lambda}{\varepsilon}\left(u-u_{e n}(t)\right) & \text { at } r=\varepsilon, t \in(0, T], \\ u=\delta_{n} & \text { at } r=n, t \in(0, T], \\ u=u_{0 n} & \text { at } t=0, r \in[\varepsilon, n],\end{cases}
$$

for $n \geqq 1$. Existence and uniqueness of solutions to this problem are classical and can be found in e.g. [12], page 491. The solutions obtained have the regularity

$$
C^{\infty}\left(S_{T}^{\varepsilon, n}\right) \cap C^{2+\alpha, 1+\alpha / 2}\left(\overline{S_{T}^{\varepsilon, n}}\right) .
$$

In order to obtain an estimate on the spatial derivative of the solutions $u_{n}$, we derive an equation for the flux

$$
F_{n}=\lambda u_{n}-r u_{n r}
$$

The functions $u_{n}$ satisfy the equation

$$
\beta^{\prime}\left(u_{n}\right) u_{n t}+\frac{1}{r} F_{n r}=0
$$

Differentiating this equation with respect to $r$ yields for $F_{n}$ the uniformly parabolic equation 


$$
\beta^{\prime}\left(u_{n}\right) F_{n t}=F_{n r r}-F_{n r}\left\{\frac{\lambda+1}{r}+\frac{\beta^{\prime \prime}\left(u_{n}\right)}{\beta^{\prime}\left(u_{n}\right)} u_{n r}\right\} \quad \text { in } S_{T}^{\varepsilon, n} .
$$

Using (2.5) once more we find the boundary conditions

$$
\begin{aligned}
F_{n}=\lambda u_{e n}, & r=\varepsilon ; \\
F_{n r}=0, & r=n .
\end{aligned}
$$

Hypothesis (A1) and properties (2.1) and (2.2) of the functions $u_{0 n}$ imply that $F_{n}$ is bounded uniformly in $n$ at $t=0$. By the maximum principle, the same then holds for $F_{n}$ on the whole of $\overline{S_{T}^{\varepsilon, n}}$. Therefore

$$
\frac{\sup _{S_{T}^{\varepsilon, n}}}{S_{n}^{\varepsilon, n}}\left|u_{n r}\right| \leqq L
$$

for some $L>0$ that is independent of $n$.

Next we investigate the regularity in time. We first consider the behaviour of $u_{n}$ at the boundary $r=\varepsilon$.

Lemma 2.2. There exists a positive constant $c$ independent of $n$ such that

$$
\left|u_{n}\left(\varepsilon, t_{2}\right)-u_{n}\left(\varepsilon, t_{1}\right)\right| \leqq c\left|t_{2}-t_{1}\right|^{\frac{1}{2}}
$$

for all $0 \leqq t_{1} \leqq t_{2} \leqq T$.

Proof. We shall only prove the inequality $u_{n}\left(\varepsilon, t_{2}\right)-u_{n}\left(\varepsilon, t_{1}\right) \geqq-c\left(t_{2}-t_{1}\right)^{1 / 2}$ for $t_{2} \geqq t_{1}$; the opposite inequality follows along the same lines.

We first consider an auxiliary problem: find $z:[0, \infty) \times[0, \infty) \rightarrow \mathbb{R}$ that satisfies

$$
z_{t}=z_{x x} \text { for all }(x, t) \in(0, \infty) \times(0, \infty) \text {, }
$$

along with initial condition $z(\cdot, 0)=0$ and boundary condition $z_{x}(0, \cdot)=1$. This problem has a unique solution which is of the form

$$
z(x, t)=\sqrt{t} f\left(\frac{x}{\sqrt{t}}\right) .
$$

It is not difficult to verify that $f$ is negative on $[0, \infty)$, has a finite limit $f(0)$, and satisfies $f^{\prime \prime}<0$ on $(0, \infty)$.

We now construct a comparison function for equation (2.4) that is based on the function $z$. For $0<b \leqq \min \left\{\beta^{\prime}(s): 0 \leqq s \leqq 1\right\}$ to be chosen later, define

$$
v(r, t)=u_{n}\left(\varepsilon, t_{1}\right)+z\left(b^{-1 / 2}(r-\varepsilon), t-t_{1}\right)-L(r-\varepsilon)-m\left(t-t_{1}\right),
$$

on the set $\varepsilon \leqq r \leqq n, t_{1} \leqq t \leqq T$, where $m=|\lambda-1|\left(b^{-1 / 2}+L\right) /(b \varepsilon)$. It then follows that 


$$
\begin{aligned}
\beta(v)_{t}-v_{r r}+\frac{\lambda-1}{r} v_{r} & =\beta^{\prime}(v)\left\{v_{t}-\frac{1}{\beta^{\prime}(v)} v_{r r}+\frac{\lambda-1}{r \beta^{\prime}(v)} v_{r}\right\} \\
& \leqq \beta^{\prime}(v)\left\{v_{t}-\frac{1}{b} v_{r r}+\frac{|\lambda-1|}{b \varepsilon}\left|v_{r}\right|\right\} \\
& \leqq 0,
\end{aligned}
$$

where we have used the fact that $v_{r r}<0$ in the first inequality. In (2.7) we have changed the nonlinearity $\beta$ outside the range of $u_{n}$ such that $\beta(v)$ is well-defined and $0<b<\beta^{\prime}(s)<\infty$ for all $s \in \mathbb{R}$. This is necessary because $v$ may not be positive everywhere on its domain.

We prove that $u_{n} \geqq v$, which implies the assertion. It follows from (2.7) that the minimum of $u_{n}-v$ is assumed on the parabolic boundary of the set $\left\{\varepsilon<r<n, t_{1}<t \leqq T\right\}$. The bound (2.6) ensures that $u_{n} \geqq v$ at $t=t_{1}$, and since $u_{n}(n, t)=1 \geqq u_{n}\left(\varepsilon, t_{1}\right)$ the same holds on the right boundary $\left\{r=n, t_{1}<t \leqq T\right\}$. Therefore a negative minimum of $u_{n}-v$ can only be assumed on the boundary $r=\varepsilon$, where

$$
\begin{aligned}
\left(u_{n}-v\right)_{r} & =\frac{\lambda}{\varepsilon}\left(u_{n}-u_{e n}(t)\right)-b^{-1 / 2} z_{r}+L \\
& \leqq \frac{\lambda}{\varepsilon}+L-b^{-1 / 2} .
\end{aligned}
$$

Choosing $b$ sufficiently small we therefore obtain $\left(u_{n}-v\right)_{r}<0$ on the boundary $r=\varepsilon$, and conclude that $u_{n} \geqq v$ on $\left\{\varepsilon \leqq r \leqq n, t_{1} \leqq t \leqq T\right\}$.

The regularity result of Gilding [6] then yields that

$$
\left\|u_{n}\right\|_{C^{0+1,0+1 / 2}\left(\overline{\left.S_{T}^{c, n}\right)}\right.} \leqq C \text { for all } n .
$$

This suffices to apply the Arzelà-Ascoli Theorem and conclude that there exists a subsequence that converges uniformly on compact subsets of $\overline{S_{T}^{\varepsilon}}$. By a familiar argument (see e.g. Oleinik [13], p. 361) the limit function $u$ can be shown to be a weak solution of Problem I. This concludes the proof of existence.

The uniqueness follows directly from this comparison principle:

Proposition 2.3 (Comparison Principle). Let $u^{1}$ be a subsolution and $u^{2}$ be a supersolution of Problem I, with initial values $u_{0}^{1}$ and $u_{0}^{2}$, and boundary conditions at $r=\varepsilon$ :

$$
u_{r}^{1} \geqq \frac{\lambda}{\varepsilon}\left(u^{1}-u_{e}^{1}\right) \quad \text { and } \quad u_{r}^{2} \leqq \frac{\lambda}{\varepsilon}\left(u^{2}-u_{e}^{2}\right) .
$$

If $u_{0}^{1} \leqq u_{0}^{2}$ on $[\varepsilon, \infty)$ and $u_{e}^{1} \leqq u_{e}^{2}$, then $u^{1} \leqq u^{2}$ on $S_{T}^{\varepsilon}$.

The proof of Proposition 2.3 is a simple extension of the proof in Goncerzewicz [11], and follows the ideas of Díaz and Kersner [3]. This completes the proof of Theorem 2.1. 
We conclude this section with a property of solutions of Problem I that is crucial for the large-time behaviour.

Proposition 2.4 (mass conservation). Let $u$ be a solution of Problem I. Then

$$
\int_{\varepsilon}^{\infty}\left\{\beta(u(r, t))-\beta\left(u_{0}(\infty)\right)\right\} r d r=\int_{\varepsilon}^{\infty}\left\{\beta\left(u_{0}(r)\right)-\beta\left(u_{0}(\infty)\right)\right\} r d r+\lambda t\left(u_{e}-u_{0}(\infty)\right) .
$$

This can be interpreted as stating that the only increase of 'mass' - in the case of the model described in the introduction, this would be mass of contaminant - comes from the injection at the boundary. The proof of this statement is analogous to the proof of mass conservation for the porous media equation (1.5) [7].

\section{Limit profiles}

In order to obtain solutions of (1.8) subject to boundary conditions (1.9), (1.10) we consider the slightly more general problem

$$
\mathrm{P}(a, b)\left\{\begin{array}{l}
\frac{1}{2} \eta^{2}\{\beta(f)\}^{\prime}+\left(\eta f^{\prime}-\lambda f\right)^{\prime}=0, \quad 0<\eta<\infty \\
f(0)=a, \quad f(\infty)=b
\end{array}\right.
$$

for any $a, b \in[0,1]$. We first prove existence and uniqueness of solutions of $\mathrm{P}(a, b)$ and then enter more deeply into the specific cases $\mathrm{P}(0,1)$ and $\mathrm{P}(1,0)$. Some of the proofs will only be sketched; the reader can find comprehensive and detailed studies of Problem $\mathrm{P}(1,0)$ in [5] and of Problem $\mathrm{P}(0,1)$ in [15].

3.1. Existence and uniqueness. Because of the possible degeneration of the equation when $f=0$, we must again define the notion of a solution of this problem. For convenience we set

$$
F(\eta)=\eta f^{\prime}(\eta)-\lambda f(\eta), \quad \eta>0
$$

Definition. Let $a, b \in[0,1]$. A function $f:[0, \infty) \rightarrow[0,1]$ is called a solution of Problem $\mathrm{P}(a, b)$ if

(i) $F$ and $\beta(f)$ are locally absolutely continuous on $[0, \infty)$;

(ii)

$$
F^{\prime}+\frac{1}{2} \eta^{2}\{\beta(f)\}^{\prime}=0 \quad \text { a.e. on }(0, \infty)
$$

$$
f(0)=a \text { and } f(\infty)=b .
$$

We can directly deduce from this definition 
Proposition 3.1. Let $f$ be a solution of Problem $\mathrm{P}(a, b)$ and let $\mathscr{P}$ be the positivity set $\{\eta>0: f(\eta)>0\}$. Then

(i) $f \in C^{1}((0, \infty)) \cap C^{\infty}(\mathscr{P})$;

(ii) $f$ is monotone, and $f^{\prime} \neq 0$ on $\mathscr{P}$ unless $a=b$;

(iii) $F(\eta) \rightarrow-\lambda b$ as $\eta \rightarrow \infty$.

Proof. Parts (i) and (iii) are proven in [5], and part (ii) follows from a local uniqueness argument as in [1], [2].

About the positivity set $\mathscr{P}$ we remark that

- if $a=0$ and $b>0$, then $\mathscr{P}=(0, \infty)[15]$;

- if $a>0$ and $b=0$, then we distinguish two cases: if $1 / \beta(s)$ is integrable at $s=0$, then $\mathscr{P}=[0, L)$ for some $L>0$; otherwise $\mathscr{P}=[0, \infty)[5]$. infinity:

When $\mathscr{P}$ is unbounded, we have an a priori estimate of the rate of convergence at

Proposition 3.2. Let $f$ be a solution of Problem $\mathrm{P}(a, b)$. Then there exist positive constants $\eta_{0}, C$, and $K$, such that

$$
|b-f(\eta)| \leqq C \int_{\frac{1}{\lambda} \eta^{\lambda}}^{\infty} e^{-K \sigma^{2 / \lambda}} d \sigma
$$

for all $\eta>\eta_{0}$.

The proof is given in [15] and uses a lower bound of $\beta^{\prime}(s)$ near $s=b$. Note that (9.3) implies that

$$
\begin{aligned}
|b-f(\eta)| & \leqq C \int_{\frac{1}{\lambda} \eta^{\lambda}}^{\infty} e^{-K \sigma^{2 / \lambda}} d \sigma \\
& \leqq C \int_{\frac{1}{\lambda} \eta^{\lambda}}^{\infty} \frac{2 K}{\lambda} \sigma^{2 / \lambda-1} e^{-K \sigma^{2 / \lambda}} d \sigma \\
& =C e^{-K \lambda-2 / j \eta^{2}},
\end{aligned}
$$

if $\eta$ is large enough.

We have the following comparison principle.

Proposition 3.3 (Comparison Principle). Let $f_{i}$, for $i=1$, 2, be solutions of $\mathrm{P}\left(a_{i}, b_{i}\right)$ with $a_{i}, b_{i} \in[0,1]$. If $a_{1} \leqq a_{2}$ and $b_{1} \leqq b_{2}$ then $f_{1} \leqq f_{2}$ on $[0, \infty)$.

Proof. Denote the positivity sets of the functions $f_{1}$ and $f_{2}$ by $\mathscr{P}_{1}$ and $\mathscr{P}_{2}$. Suppose that the difference $v=f_{1}-f_{2}$ assumes a positive maximum at $\eta_{0} \in(0, \infty)$. Then $f_{1}\left(\eta_{0}\right)>0$ so that $\eta_{0} \in \mathscr{P}_{1}$, which implies that $f_{1}$ is twice continuously differentiable in $\eta_{0}$. 
- If $\eta_{0} \in \mathscr{P}_{2}$ then $f_{2}$ also is twice differentiable in $\eta_{0}$, and then the result follows from subtracting the equations for $f_{1}$ and $f_{2}$ at $\eta=\eta_{0}$.

- If $\eta_{0} \in \mathbb{R}^{+} \backslash \mathscr{P}_{2}$ then $f_{2}^{\prime}\left(\eta_{0}\right)=0$, which implies $f_{1}^{\prime}\left(\eta_{0}\right)=0$. From Proposition 3.1 it follows that this only is possible when $a_{1}=b_{1}$ and $f_{1}$ is constant on $\mathscr{P}_{1}$. Because $f_{1}\left(\eta_{0}\right)>0$ we have $a_{1}=b_{1}>0$, and the boundary conditions then imply that $f_{2}$ is not monotone. This contradicts Proposition 3.1.

Corollary 3.4. For every $a, b \in[0,1]$, Problem $\mathrm{P}(a, b)$ has at most one solution.

Proposition 3.5. For every $a, b \in[0,1]$, Problem $\mathrm{P}(a, b)$ has a solution (which is unique by Corollary 3.4). be written as

Proof. With the change of variables $s=\frac{1}{\lambda} \eta^{\lambda}$ and $g(s)=f(\eta)$, Problem $\mathrm{P}(a, b)$ can
ritten as

$$
P_{g}(a, b)\left\{\begin{array}{l}
g^{\prime \prime}+\mu s^{\alpha}\{\beta(g)\}^{\prime}=0 \text { for } 0<s<\infty, \\
g(0)=a, \quad g(\infty)=b,
\end{array}\right.
$$

where $^{\prime}=d / d s$ and the constants $\alpha$ and $\mu$ are given by

$$
\alpha=\frac{2}{\lambda}-1 \quad \text { and } \quad \mu=\frac{1}{2} \lambda^{\alpha}
$$

A solution of $\mathrm{P}_{g}(a, b)$ is defined in a sense similar to the case of Problem $\mathrm{P}(a, b)$, and it can easily be verified that the two problems are equivalent.

In both $a$ and $b$ are positive, then by the Comparison Principle any solution of Problem $\mathrm{P}(a, b)$ will take values between $a$ and $b$. Therefore the problem is non-degenerate and the existence of a solution to the boundary value problem $\mathrm{P}(a, b)$ can be shown by a shooting argument: if $h$ is the solution of (3.5) with initial conditions $h(0)=a$ and $h^{\prime}(0)=A$, then $\lim _{s \rightarrow \infty} h(s)$ exists for all $A>0$, depends continuously on $A$, and tends to zero or infinity when $A \rightarrow 0$ or $A \rightarrow \infty$. This implies that there exists an $A$ such that the limit is equal to $b$. The details of this argument can be found in [15] and a similar argument is used by Gilding and L.A. Peletier ([9], p. 532). In the rest of this proof we will suppose that $a=0$ and $b>0$, and merely assert that the other case, $b=0$ and $a>0$, can be handled in an analogous way.

A solution of $\mathrm{P}_{g}(0, b)$ is constructed as the limit as $\varepsilon \downarrow 0$ of solutions of $\mathrm{P}_{g}(\varepsilon, b)$. For $\varepsilon>0$ the solution of $\mathrm{P}_{g}(\varepsilon, b)$ is defined and unique, and by the Comparison Principle the sequence $\left\{g_{\varepsilon}\right\}$ depends monotonically on $\varepsilon$. We now show that the pointwise limit of this sequence, denoted by $g$, is a solution of Problem $\mathrm{P}_{g}(a, b)$. By twice integrating the equation in $\mathrm{P}_{g}(\varepsilon, b)$ we find the following integral identity for $g_{\varepsilon}$ :

$$
g_{\varepsilon}(s)=b-\mu \int_{s}^{\infty}[(1+\alpha) \sigma-\alpha s] \sigma^{\alpha-1}\left\{\beta(b)-\beta\left(g_{\varepsilon}(\sigma)\right)\right\} d \sigma
$$

for all $s \in[0, \infty)$. The finiteness of the integral follows from the exponential convergence proved in Proposition 3.2. Since $g_{\varepsilon} \downarrow g$ as $\varepsilon \rightarrow 0$, and therefore $\left(\beta(b)-\beta\left(g_{\varepsilon}\right)\right) \uparrow(\beta(b)-\beta(g))$ 
on $[0, \infty)$, we can apply the monotone convergence theorem to the integral in (3.6) to conclude that it converges; the positivity of the left-hand side implies that the limit is finite. This results in the same integral equality for the limit function $g$ :

$$
g(s)=b-\mu \int_{s}^{\infty}[(1+\alpha) \sigma-\alpha s] \sigma^{\alpha-1}\{\beta(b)-\beta(g(\sigma))\} d \sigma
$$

for all $s \in[0, \infty)$. Starting with (3.7) and differentiating twice we can show that $g$ is a solution to Problem $\mathrm{P}_{g}(0, b)$. This implies that the corresponding function $f$ is a solution of Problem $\mathrm{P}(0, b)$.

3.2. Behaviour near zero. In the proofs of Section 4 we need an estimate of the behaviour of the similarity solution near the origin. We restrict ourselves to the cases $P(0,1)$ and $\mathrm{P}(1,0)$.

Proposition 3.6. Let $f$ be the solution of Problem $\mathrm{P}(1,0)$. Then

$$
\lim _{\eta \downarrow 0} \eta^{1-\lambda} f^{\prime}(\eta) \text { exists in }(-\infty, 0) \text {. }
$$

Proof. Writing equation (3.1) in the form

$$
\frac{f^{\prime \prime}}{f^{\prime}}=\frac{\lambda-1}{\eta}-\frac{1}{2} \eta \beta^{\prime}(f(\eta))
$$

we obtain for arbitrary $\eta, \eta_{0} \in \mathscr{P}$,

$$
\eta^{1-\lambda} f^{\prime}(\eta)=\eta_{0}^{1-\lambda} f^{\prime}\left(\eta_{0}\right) e^{-\frac{1}{2} \int_{n_{0}}^{\eta} y \beta^{\prime}(f(y)) d y}
$$

Letting $\eta \downarrow 0$ yields the result.

For $\mathrm{P}(0,1)$ the analysis is more involved because the degeneracy of the nonlinearity and the geometric degeneracy coincide at $\eta=0$. We encounter these two elements when describing the behaviour of solutions. In order to be able to make definite statements we must assume the extra hypothesis on $\beta$

$$
\left(\mathrm{H}_{\beta} 3\right) \quad \lim _{f \downarrow 0} \frac{f \beta^{\prime \prime}(f)}{\beta^{\prime}(f)}=p-1 \text { for some constant } p \in(0,1] .
$$

This condition expresses that for small data $\beta$ behaves essentially as a power with exponent $p$.

For a nonlinearity $\beta$ in the form of $\beta(f)=c f^{p}$, equation (3.1) has certain scaling properties that allow us to transform it into an autonomous one, and then apply a phase plane analysis. This analysis, which contains a complete classification of the behaviour of solutions near the origin, is given in [15]. Here we summarise the results.

Proposition 3.7. Let $f$ be the solution of $\mathrm{P}(0,1)$, where $\beta(f)=c f^{p}$ for some $p \in(0,1)$ and $c>0$, and let $\mu$ be given, as in Proposition 3.5, by $\mu=\frac{1}{2} \lambda^{\frac{2}{\lambda}-1}$. 
(a) If $\lambda<2 /(1-p)$, then the limit

$$
\lim _{\eta \downarrow 0} \frac{f(\eta)}{\eta^{\lambda}} \text { exists in }(0, \infty)
$$

(b) If $\lambda=2 /(1-p)$, then

$$
\lim _{\eta \downarrow 0} \frac{f^{1-p}(\eta)}{\eta^{2} \log \eta}=-2 c \mu p \lambda^{1-p}
$$

(c) If $\lambda>2 /(1-p)$, then

$$
\lim _{\eta \downarrow 0} \frac{f(\eta)}{\eta^{\frac{2}{1-p}}}=\frac{A}{\lambda^{k}}
$$

in which $k=2 /(\lambda(1-p))$ and

$$
A=\left(\frac{c \mu p}{1-k}\right)^{\frac{1}{1-p}}
$$

For more general nonlinearities $\beta$ the analysis is more involved, and the results less precise. We find Then:

Proposition 3.8. Let $f$ be the solution of Problem $\mathrm{P}(0,1)$, where $\beta$ satisfies $\left(\mathrm{H}_{\beta} 1-3\right)$.

(a) If $\lambda<\frac{2}{1-p}$, then $\lim _{\eta \downarrow 0} \eta^{-\lambda} f(\eta)$ exists in $(0, \infty)$.

(b) If $\lambda \geqq \frac{2}{1-p}$, then $\lim _{\eta \downarrow 0} \eta^{2} \beta^{\prime}(f(\eta))=2\left(\lambda-\frac{2}{1-p}\right)$.

The number $2 /(1-p)$ should be replaced by $\infty$ when $p=1$.

Proof. Introducing the variables

$$
\gamma(s)=\frac{s g^{\prime}(s)}{g(s)} \quad \text { and } \quad \delta(s)=\mu s^{\alpha+1} \beta^{\prime}(g(s))
$$

in equation (3.5), we find that they satisfy the system of equations

$$
\left\{\begin{array}{l}
s \gamma^{\prime}=\gamma(1-\gamma-\delta) \\
s \delta^{\prime}=\delta(\alpha+1+\zeta(s) \gamma)
\end{array}\right.
$$

where

$$
\zeta(s)=\frac{g(s) \beta^{\prime \prime}(g(s))}{\beta^{\prime}(g(s))} .
$$

By $\left(\mathrm{H}_{\beta} 3\right)$ and the boundary condition $g(0)=0$ we observe that $\zeta(s) \rightarrow p-1$ as $s \downarrow 0$. Consequently this system is asymptotically autonomous in the sense of Thieme [17] as 
$s \downarrow 0$ (or if $\sigma=\log s$, as $\sigma \rightarrow-\infty$ ). We wish to apply a theorem of Poincaré-Bendixson type (Theorem 1.6 of the same reference) to conclude that $(\gamma, \delta)$ tends to an equilibrium of the 'limit' system

$$
\left\{\begin{array}{l}
s \gamma^{\prime}=\gamma(1-\gamma-\delta) \\
s \delta^{\prime}=\delta(\alpha+1+(p-1) \gamma)
\end{array}\right.
$$

as $s \downarrow 0$. According to [17], the only remaining condition to be verified is that the orbit under consideration is bounded as $s \downarrow 0$.

To show that this is the case, remark that the concavity of $g$ implies that

$$
g(s) \geqq s g^{\prime}(s) \text { for all } s>0,
$$

which gives $0 \leqq \gamma \leqq 1$ for all $s>0$. Since $\delta$ is positive, the orbit $(\gamma, \delta)$ can only be unbounded in the positive $\delta$-direction. To force a contradiction, suppose that there exists a sequence $s_{n} \downarrow 0$ such that $\delta\left(s_{n}\right) \rightarrow \infty$ and such that $\delta^{\prime}\left(s_{n}\right)<0$ and $\left|\delta^{\prime}\left(s_{n}\right) / \gamma^{\prime}\left(s_{n}\right)\right| \rightarrow \infty$. Since $\alpha+1>0$ and $\zeta(s) \rightarrow p-1$ when $s \downarrow 0$, there exists an $\varepsilon>0$ such that $\delta^{\prime}$ is positive when $\gamma<\varepsilon$. It therefore follows that $\gamma\left(s_{n}\right) \geqq \varepsilon$. On the other hand, we can write

$$
\frac{\delta^{\prime}}{\gamma^{\prime}}=\frac{\delta(\alpha+1+\zeta(s) \gamma)}{\gamma(1-\gamma-\delta)}
$$

and if $\gamma \geqq \varepsilon$ then the right-hand side of this expression is bounded from above and below when $\delta$ is large. This contradicts the assumption that $\left|\delta^{\prime}\left(s_{n}\right) / \gamma^{\prime}\left(s_{n}\right)\right| \rightarrow \infty$ as $n \rightarrow \infty$, and we conclude that the orbit $(\gamma, \delta)$ is bounded and therefore tends to an equilibrium of the limit system (3.9).

For the analysis of the equilibrium points of (3.9) it is convenient to introduce

Definition. Let $\varphi \in C^{1}(0, \delta)$ for some $\delta>0$. Then

$$
v(\varphi) \stackrel{\text { def }}{=} \lim _{x \downarrow 0} \frac{x \varphi^{\prime}(x)}{\varphi(x)} \quad \text { (provided this limit exists) }
$$

is called the index of $\varphi$.

If $\varphi$ is a power of its argument, $v(\varphi)$ simply is the exponent. One can derive some properties of $v$ which extend this correspondance: if $\varphi$ and $\psi$ are such that $v(\varphi)$ and $v(\psi)$ are defined, then

(i) $\quad v(\varphi \psi)=v(\varphi)+v(\psi)$;

(ii) $v(\varphi \circ \psi)=v(\varphi) v(\psi)$ provided $\psi(0)=0$;

(iii) $v\left(\varphi^{\alpha}\right)=\alpha \nu(\varphi)$ for all $\alpha \in \mathbb{R}$;

(iv) $\nu(\varphi)>-1 \Rightarrow \varphi \in L^{1}(0, \delta)$. 
Besides, by de l'Hôpital's rule, the existence of $v\left(\varphi^{\prime}\right)$ implies that $v(\varphi)$ exists and that

(v) $v(\varphi)=1+v\left(\varphi^{\prime}\right)$.

Note that with this notation assumption $\left(\mathrm{H}_{\beta} 3\right)$ can be written as $v\left(\beta^{\prime}\right)=p-1$. the point

The system (3.9) has the equilibria $e_{0}=(0,0)$ and $e_{1}=(1,0)$, and if $\alpha+p<0$ then

$$
e_{2}=\left(\frac{\alpha+1}{1-p},-\frac{\alpha+p}{1-p}\right)
$$

is also an equilibrium point. Of these equilibria the first, $(0,0)$, can be quickly ruled out: by definition $v(g)=\lim _{s \downarrow 0} \gamma(s)$, and by writing equation (3.5) as

$$
\frac{s g^{\prime \prime}(s)}{g^{\prime}(s)}=-\delta(s)
$$

we see that $v\left(g^{\prime}\right)=-\lim _{s \downarrow 0} \delta(s)$. Consequently $(\gamma, \delta) \rightarrow(0,0)$ implies on one hand $v\left(g^{\prime}\right)=0$ and on the other hand $v(g)=0$; this is incompatible by property (v) above. For the other two equilibria, we distinguish three cases:

- when $\alpha+p<0$, the equilibrium $(1,0)$ is unstable (in backward time) and is therefore not admissible; it follows that $(\gamma, \delta) \rightarrow e_{2}$ as $s \downarrow 0$, and more specifically

$$
\delta(s) \rightarrow-(\alpha+p) /(1-p) ;
$$

- when $\alpha+p=0, e_{1}=e_{2}$ and therefore $\delta(s) \rightarrow 0$ as $s \downarrow 0$;

- when $\alpha+p>0, e_{1}$ is the only admissible equilibrium and therefore $v(g)=1$; using properties (i)-(iii) we find that

$$
v\left(s^{\alpha} \beta^{\prime}(g(s)) g^{\prime}(s)\right)=\alpha+p-1>-1,
$$

which implies by (3.5) and property (iv) that $g^{\prime \prime}$ is integrable; as a result, $g^{\prime}(0+)$ is finite.

We can rearrange this information in the following form:

- when $\alpha+p>0, \lim _{s \downarrow 0} g^{\prime}(s)$ is finite;

- when $\alpha+p \leqq 0, \lim _{s \downarrow 0} \mu s^{\alpha+1} \beta^{\prime}(g(s))=-\frac{\alpha+p}{1-p}$.

In terms of $\lambda, f$, and $\eta$, this is the statement of the theorem.

\section{The main result}

This section is devoted to the proofs of Theorems A and B. We shall discuss the proof in full for Theorem A, and merely comment on the differences with Theorem B. 
Proof of Theorem A. In order to compare the solution of the original problem with the self-similar solution we reformulate the problem in self-similar variables. If $u$ is the solution of Problem I, then define $z$ by $z(\eta, \tau)=u(r, t)$, where the independent variables are again linked through the relations

$$
\eta=\frac{r}{\sqrt{t}} \text { and } \tau=\frac{t}{\varepsilon^{2}}
$$

The function $z$ satisfies the equation

$$
\tau \beta(z)_{\tau}-\frac{1}{2} \eta \beta(z)_{\eta}+\frac{\lambda-1}{\eta} z_{\eta}-z_{\eta \eta}=0 \text { for } \eta>1 / \sqrt{\tau}, \tau>0
$$

and the boundary condition

$$
z_{\eta}=\lambda \sqrt{\tau}(z-1) \text { for } \eta=1 / \sqrt{\tau}, \tau>0
$$

The first step consists of an integral estimate, derived from the differential equation.

Proposition 4.1. Let $\Phi: \mathbb{R}^{+} \rightarrow \mathbb{R}$ be defined by

$$
\Phi(\tau)=\int_{1 / V_{\tau}}^{\infty} \eta\{\beta(z(\eta, \tau))-\beta(f(\eta))\} d \eta, \quad \tau>0
$$

and suppose that either $\varepsilon$ is fixed or $u_{0}$ is constant. Then there is a constant $\chi$ such that

$$
\Phi(\tau) \leqq \frac{\chi}{\tau} \quad \text { for all } \tau>0
$$

If $u_{0}$ is constant, then $\chi$ does not depend on $\varepsilon$.

Proof. By integrating (1.8) and using boundary conditions (1.9) and Proposition 3.2 we find that

$$
\int_{1 / \sqrt{\tau}}^{\infty} \beta(f(\eta)) \eta d \eta=\lambda-\int_{0}^{1 / \sqrt{\tau}} \beta(f(\eta)) \eta d \eta
$$

The conservation of mass (Proposition 2.4) reads in the $\eta, \tau$ coordinates

$$
\int_{1 / V_{\tau}}^{\infty} \beta(z) \eta d \eta=\frac{1}{\varepsilon^{2} \tau} \int_{\varepsilon}^{\infty} \beta\left(u_{0}(r)\right) r d r+\lambda
$$

By combining these two we find that $\Phi$ is well-defined and that

$$
\tau \Phi(\tau)=\frac{1}{\varepsilon^{2}} \int_{\varepsilon}^{\infty} \beta\left(u_{0}(r)\right) r d r+\tau \int_{0}^{1 / V_{\tau}} \beta(f(\eta)) \eta d \eta .
$$


The second term in (4.3) is bounded by $\beta(1) / 2$. When $\varepsilon$ is constant, the result follows immediately; when $\varepsilon$ varies, but $u_{0}$ is constant and therefore equal to 0 , the first term on the right-hand side vanishes and the remainder is bounded independent of $\varepsilon$.

The interest of this integral estimate lies in the fact that $z$ and $f$ are ordered, and that therefore the argument of the integral is positive. Indeed, if $v$ is the self-similar solution of equation (Ia) corresponding to $f$, i.e. $v(r, t)=f(r / \sqrt{t})$, we can integrate equation (I a) from 0 to $\varepsilon$ to obtain

$$
\int_{0}^{\varepsilon} \beta(v)_{t} r d r+\left[\lambda v-r v_{r}\right]_{0}^{\varepsilon}=0
$$

Now $v_{t}(r, t)=-\frac{1}{2} r t^{-3 / 2} f^{\prime}(r / \sqrt{t})>0$ for all $r$ and $t$ and therefore we have

$$
\lambda v(\varepsilon, t)-\varepsilon v_{r}(\varepsilon, t) \leqq \lambda .
$$

By the Comparison Principle (Proposition 2.3) we then find that $u$ lies above $v$ on the whole of $S_{T}^{\varepsilon}$, which implies the same for $z$ and $f$ (on the appropriate domain).

Our aim is to convert an integral estimate related to (4.2) into a pointwise estimate. For this we need the next lemma (for an idea of the proof we refer to [14]).

Lemma 4.2. Let $\phi$ be a non-negative continuous function on $[0, \infty)$ with lower Lipschitz constant L, i.e.

$$
\frac{\phi(x)-\phi(y)}{x-y} \geqq-L \text { for all } x, y \in[0, \infty), x \neq y \text {. }
$$

Let $x_{0}>0$. If $\int_{x_{0}}^{\infty} x \phi(x) d x \leqq \alpha$, then

$$
\sup _{x_{0} \leqq x<\infty} \phi(x) \leqq \sqrt[3]{6 L^{2} \alpha} .
$$

We shall not apply this lemma directly to $\Phi$, but to the integral

$$
\int_{1 / V_{\tau}}^{\infty} \eta\{z(\eta, \tau)-f(\eta)\} d \eta
$$

For this integral we obtain an estimate similar to (4.2) by pointing out that, because $\beta$ is concave and strictly increasing on its domain, the function $s \mapsto \beta(s)-\beta^{\prime}(1) s$ is nondecreasing for $0 \leqq s \leqq 1$. This implies that $\beta(z(\eta, \tau))-\beta(f(\eta)) \geqq \beta^{\prime}(1)(z(\eta, \tau)-f(\eta))$ and thus

$$
\int_{1 / \sqrt{\tau}}^{\infty} \eta\{z(\eta, \tau)-f(\eta)\} d \eta \leqq \frac{\chi}{\beta^{\prime}(1) \tau}
$$

The crucial part in the application of Lemma 4.2 to estimate (4.4), with

$$
\phi(\eta, \tau)=z(\eta, \tau)-f(\eta)
$$

7 Journal für Mathematik. Band 479 
is that we need to verify the lower Lipschitz continuity of $\phi$ with respect to the variable $\eta$. For general $\beta$, the function $\beta(z(\eta, \tau))$ need not be lower Lipschitz continuous with respect to $\eta$, and therefore we switched here from (4.2) to (4.4). From Proposition 3.1 we know that $f$ is nonincreasing on $\mathbb{R}^{+}$, so the lower Lipschitz constant of $\phi$ only depends on $z$. We have

Proposition 4.3. If $0<\lambda<1$, then there exist positive constants $\ell$ and $m$ independent of $\eta$ and $\tau$ such that

$$
z_{\eta}(\eta, \tau) \geqq-\ell \tau^{\frac{1-\lambda}{2}}-m \text { for all } \tau>0 \text { and } \eta>1 / \sqrt{\tau} \text {. }
$$

If $\lambda \geqq 1$, then there exists a constant $m$ independent of $\eta$ and $\tau$ such that

$$
z_{\eta}(\eta, \tau) \geqq-m \text { for all } \tau>0 \text { and } \eta>1 / \sqrt{\tau} \text {. }
$$

If, for the moment, we consider this proposition proved, the conclusions of Theorem A follow by combining (4.4) and Proposition 4.3 and applying Lemma 4.2.

Proof of Proposition 4.3. Let $z_{n}(\eta, \tau)=u_{n}(r, t)$ where $u_{n}$ denotes the regularised solution constructed in Section 2. The domain of definition of $z_{n}$ is

$$
D_{T}^{\varepsilon, n}=\left\{(\eta, \tau): \frac{1}{\sqrt{\tau}}<\eta<\frac{n}{\varepsilon \sqrt{\tau}}, 0<\tau \leqq \frac{T}{\varepsilon^{2}}\right\}
$$

which is drawn in Figure 4.1.

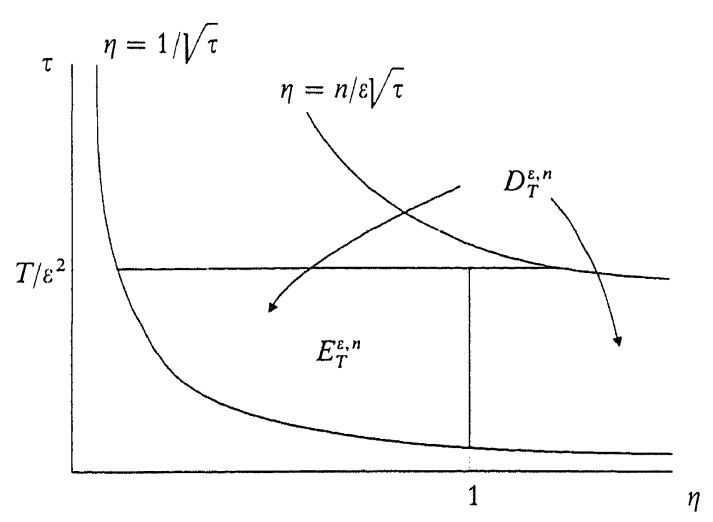

Figure 4.1. The domains $D_{T}^{\varepsilon, n}$ and $E_{T}^{\varepsilon, n}$

The first part of the proof is the following lemma.

Lemma 4.4. There exists a positive constant $C$, depending on $\beta, \lambda$ and $u_{0}$, such that

$$
\left|z_{n \eta}\right| \leqq \frac{C}{\eta} \quad \text { on } D_{T}^{\varepsilon, n} .
$$

The constant $C$ does not depend on $n$ or $T$, and if $u_{0}$ is constant, then it does not depend on $\varepsilon$, either. 
Proof of Lemma 4.4. We use again the flux $F_{n}$ introduced in the proof of Theorem 2.1. First note that

$$
F_{n}(r, t)=\lambda z_{n}(\eta, \tau)-\eta z_{n \eta}(\eta, \tau)
$$

on the relevant domains. Then the estimate follows from the observation that both $F_{n}$ and $z_{n}$ are bounded uniformly in $n$. If $u_{0}=0$, then by choosing $\delta_{n}=1 / n$ in (2.3) the constant $C$ can be chosen independently of $\varepsilon$ as well.

The remainder of the proof is based on the application of the maximum principle for parabolic equations to certain flux-type functions, depending on the value of $\lambda$. We distinguish two cases.

Case I. $0<\lambda \leqq 2$. We truncate the unbounded domain $D_{T}^{\varepsilon, n}$ by considering

$$
E_{T}^{\varepsilon, n}=D_{T}^{\varepsilon, n} \cap\{\eta<1\}
$$

We assume that $n>\sqrt{T}$, so that the domain $E_{T}^{\varepsilon, n}$ is as is shown in Figure 4.1. On $E_{T}^{\varepsilon, n}$ we define the modified flux function

$$
F_{n}=F_{n}(\eta, \tau)=\eta^{1-\lambda}\left[z_{n \eta}(\eta, \tau)+\frac{1}{2} \eta \beta\left(z_{n}(\eta, \tau)\right)\right] .
$$

Using equation (4.1) we find that $F_{n}$ satisfies

$$
\tau \beta^{\prime} F_{n t}-F_{n \eta \eta}-b F_{n \eta}-c F_{n}=d \text { on } E_{T}^{\varepsilon, n},
$$

where the coefficients $b, c$, and $d$ are given by

$$
\begin{gathered}
b(\eta, \tau)=-\frac{1-\lambda}{\eta}+\frac{1}{2} \eta \beta^{\prime}-\frac{\beta^{\prime \prime}}{\beta^{\prime}} z_{n \eta}, \quad c(\eta, \tau)=\frac{1}{2}(\lambda-2)\left\{\beta^{\prime}-\frac{\beta^{\prime \prime}}{\beta^{\prime}} \beta\left(z_{n}\right)\right\}, \\
d(\eta, \tau)=\frac{1}{2}(\lambda-2) \eta^{2-\lambda} \frac{\beta^{\prime \prime}}{\beta^{\prime}} \beta\left(z_{n}\right)^{2} .
\end{gathered}
$$

Here we note $\beta^{\prime}$ and $\beta^{\prime \prime}$ for $\beta^{\prime}\left(z_{n}\right)$ and $\beta^{\prime \prime}\left(z_{n}\right)$. Due to the regularisation, the coefficients in (4.6) are all smooth and bounded on $E_{T}^{\varepsilon, n}$. Note that $c \leqq 0$ and $d \geqq 0$, and that therefore $F_{n}$ is a supersolution for the equation

$$
\tau \beta^{\prime} G_{\tau}-G_{\eta \eta}-b G_{\eta}-c G=0 \text { on } E_{T}^{\varepsilon, n} .
$$

By the maximum principle (see e.g. [16]), a non-positive minimum of $F_{n}$ on $E_{T}^{\varepsilon, n}$ must be assumed on its parabolic boundary, i.e. $\Gamma_{1} \cup \Gamma_{2}$.

On $\Gamma_{1}$, given by $\eta=1 / \sqrt{\tau}$, we use the boundary condition and find

$$
\begin{aligned}
F_{n}(1 / \sqrt{\tau}, \tau) & =\tau^{(\lambda-1) / 2}\left\{\lambda \sqrt{\tau}\left(z_{n}(1 / \sqrt{\tau}, \tau)-1\right)+\frac{1}{2} \beta\left(z_{n}\right) / \sqrt{\tau}\right\} \\
& \geqq-\lambda \tau^{\lambda / 2}(1-f(1 / \sqrt{\tau})) \\
& \geqq A,
\end{aligned}
$$


in which $A$ is the (negative) limit value from Proposition 3.6. On $\Gamma_{2}$, where $\eta=1$, we have $F_{n}=z_{n \eta}(1, \tau)+\frac{1}{2} \beta\left(z_{n}(\eta, 1)\right) \geqq-C-\frac{1}{2} \beta(1)$ by Lemma 4.4. This implies that

$$
F_{n} \geqq-\ell:=\min \left\{A,-C-\frac{1}{2} \beta(1)\right\}
$$

on $E_{T}^{\varepsilon, n}$ where $\ell>0$, and therefore we have $z_{n \eta} \geqq-\ell \eta^{\lambda-1}-\frac{1}{2} \beta(1)$ on $E_{T}^{\varepsilon, n}$ for all $n>\sqrt{T}$. When we combine this with Lemma 4.4 we obtain the required result.

Case II. The case of $\lambda>2$ demands a different modified flux function:

$$
F_{n}=F_{n}(\eta, \tau)=\eta^{1-\lambda}\left[z_{n \eta}(\eta, \tau)-\frac{1}{2} \lambda \eta\left(\beta(1)-\beta\left(z_{n}(\eta, \tau)\right)\right)\right],
$$

which satisfies

$$
\begin{aligned}
\tau \beta^{\prime} F_{n t}-F_{n \eta \eta}-b F_{n \eta}= & c\left\{F_{n}+\frac{1}{2} \lambda \eta^{2-\lambda}\left[\beta(1)-\beta\left(z_{n}\right)\right]\right\} \\
& +d\left\{F_{n}+(\lambda-1) \eta^{2-\lambda}\left[\beta(1)-\beta\left(z_{n}\right)\right]\right\} \text { on } E_{T}^{\varepsilon, n},
\end{aligned}
$$

in which the coefficients $b, c$ and $d$ are given by

$$
\begin{gathered}
b(\eta, \tau)=-\frac{1-\lambda}{\eta}+\frac{1}{2} \eta \beta^{\prime}-\frac{\beta^{\prime \prime}}{\beta^{\prime}} z_{n \eta}, \quad c(\eta, \tau)=\frac{1}{2} \lambda(\lambda-2) \frac{\beta^{\prime \prime}}{\beta^{\prime}}\left[\beta(1)-\beta\left(z_{n}\right)\right], \\
d(\eta, \tau)=-\frac{1}{2} \lambda \beta^{\prime} .
\end{gathered}
$$

Now define the function $\omega$ by

$$
\omega(F, \eta, \tau) \stackrel{\text { def }}{=} c\left\{F+\frac{1}{2} \lambda \eta^{2-\lambda}\left[\beta(1)-\beta\left(z_{n}\right)\right]\right\}+d\left\{F+(\lambda-1) \eta^{2-\lambda}\left[\beta(1)-\beta\left(z_{n}\right)\right]\right\},
$$

and remark that $c, d<0$. We claim that the function $\eta \mapsto \eta^{2-\lambda}\left(\beta(1)-\beta\left(z_{n}\right)\right)$ is bounded on $\mathbb{R}^{+}$by a constant independent of $\tau$ and $n$ : on one hand,

$$
\begin{aligned}
0 \leqq \eta^{2-\lambda}\left[\beta(1)-\beta\left(z_{n}\right)\right] & \leqq \eta^{2-\lambda}[\beta(1)-\beta(f(\eta))] \\
& \leqq 2 \beta^{\prime}(1) \frac{A}{\lambda} \eta^{2},
\end{aligned}
$$

if $\eta$ is small enough, in which $A$ is again the limit value from Proposition 3.6. On the other hand,

$$
0 \leqq \eta^{2-\lambda}\left[\beta(1)-\beta\left(z_{n}\right)\right] \leqq \beta(1) \eta^{2-\lambda} .
$$

The combination of the first for small $\eta$ and the second for large $\eta$ yields the uniform bound. Therefore, by choosing $F_{0} \in \mathbb{R}, F_{0}<A$ negative and large enough, $\omega\left(F_{0}, \eta, \tau\right)$ can 
be made positive for all $\eta$ and $\tau$. This implies that the constant $F_{0}$ is a subsolution for equation (4.8), and by following the same line of reasoning as for case I, we can conclude that $F_{n} \geqq F_{0}$ on $E_{T}^{\varepsilon, n}$, for all $n>\sqrt{T}$. The required result is then obtained in a similar fashion.

This concludes the proof of Theorem A.

The proof of Theorem B follows the same lines, with some alterations. First, the ordering of the solution $u$ and the self-similar solution $f$ is reversed, which gives rise to the definition

$$
\Phi(\tau)=\int_{1 / V_{\bar{\tau}}}^{\infty} \eta\{\beta(f(\eta))-\beta(z(\eta, \tau))\} d \eta, \quad \tau>0
$$

The assertion of Proposition 4.1, however, holds unchanged, as does its proof. Second, if we denote $f(\eta)-z(\eta, \tau)$ by $\phi(\eta, \tau)$, the application of Lemma 4.2 requires an estimate of $\phi_{\eta}$ from below. From Section 3 we know that $f$ is strictly increasing on $\mathbb{R}^{+}$. For an upper bound on $z_{\eta}$, we have

Proposition 4.5. Let $z$ be the solution of Problem I with $u_{e}=0$ and $u_{0}(\infty)=1$, transported to the $\eta, \tau$-plane. If $0<\lambda<1$ then there exist positive constants $\ell$ and $m$ independent of $\eta$ and $\tau$ such that

$$
z_{\eta}(\eta, \tau) \leqq \ell \tau^{\frac{1-\lambda}{2}}+m \text { for all } \tau>0 \text { and } \eta>1 / \sqrt{\tau}
$$

if $\lambda \geqq 1$ then there exists a constant $m$ independent of $\eta$ and $\tau$ such that

$$
z_{\eta}(\eta, \tau) \leqq m \text { for all } \tau>0 \text { and } \eta>1 / \sqrt{\tau}
$$

The proof of Proposition 4.5 follows the same lines as that of Proposition 4.3, and we shall only mention the flux function that is used:

$$
F_{n}=\eta^{1-\gamma}\left[z_{n \eta}+\frac{1}{2} \eta \beta\left(z_{n}\right)\right]
$$

where $\gamma=\min \{\lambda, 2\}$. The result is then again reached by combination of Propositions 4.1 and 4.5 and Lemma 4.2 .

\section{References}

[1] F.V.Atkinson and L. A. Peletier, Similarity profiles of flows through porous media, Arch. Rat. Mech. Anal. 42 (1971), 369-379.

[2] F.V.Atkinson and L.A. Peletier, Similarity solutions of the nonlinear diffusion equation, Arch. Rat. Mech. Anal. 54 (1974), 373-392.

[3] J.I. Díaz and R. Kersner, On a nonlinear degenerate parabolic equation in infiltration or evaporation through a porous medium, J. Diff. Equ. 69 (1987), 368-403.

[4] C.J. van Duijn and P. Knabner, Travelling waves in the transport of reactive solutes through porous media: adsorption and binary ion exchange - Parts 1 and 2, Transp. Porous Media 8 (1992), 167-225.

[5] C.J. van Duijn and P. Knabner, Flow and reactive transport in porous media induced by well injection: Similarity solution, IMA J. Appl. Math. 52 (1994), 177-200. 
[6] B.H. Gilding, Hölder continuity of solutions of parabolic equation, J. London Math. Soc. (2) 13 (1976), $103-106$.

[7] B.H. Gilding, Properties of solutions of an equation in the theory of infiltration, Arch. Rat. Mech. Anal. 65, No. 3 (1977), 203-225.

[8] B.H. Gilding, Improved theory for a nonlinear degenerate parabolic equation, Ann. Sc. Norm. Sup. Pisa Cl. Sci. IV, 16 (1989), 165-224

[9] B.H. Gilding and L.A. Peletier, On a class of similarity solutions of the porous media equation, J. Math. Anal. Appl. 57, No. 3 (1977), 522-538.

[10] J. Goncerzewicz, Properties of solutions to the initial-boundary value problem for a porous media-type equation, Math. Meth. Appl. Sci. 15 (1992), 299-314.

[11] J. Goncerzewicz, On an initial-boundary value problem for a certain class of degenerate parabolic equations, to appear.

[12] O.A. Ladyženskaja, V.A. Solonnikov and N.N. Ural'ceva, Linear and Quasi-linear Equations of Parabolic Type, Transl. Math. Monogr., American Mathematical Society, 1968.

[13] O. A. Oleinik, On some degenerate parabolic equations, Seminari dell' Istituto Nationale di Alta Matematica 1962-63, Oderisi, Gubbio (1964), 355-371.

[14] L. A. Peletier, Asymptotic behaviour of solutions of the porous media equation, SIAM J. Appl. Math. 21, No. 4 (1971), 542-551.

[15] M.A. Peletier, Similarity solutions for a nonlinear transport equation, Report 93-42 of Delft University of Technology, 1993.

[16] M.H. Protter and H.F. Weinberger, Maximum Principles in Differential Equations, Prentice-Hall, Englewood Cliffs, New Jersey.

[17] H.R. Thieme, Convergence results and a Poincaré-Bendixson trichotomy for asymptotically autonomous differential equations, J. Math. Biol. 30 (1992), 755-763.

Centrum voor Wiskunde en Informatica, PO Box 94079, NL-1090 GA Amsterdam

Eingegangen 28. Februar 1995, in revidierter Fassung 29. Januar 1996 